\title{
Data Association in a World Model for Autonomous Systems
}

\author{
Marcus Baum ${ }^{1}$, Ioana Gheţa ${ }^{2}$, Andrey Belkin², Jürgen Beyerer ${ }^{2,3}$, Uwe D. Hanebeck ${ }^{1}$ \\ ${ }^{1}$ Karlsruhe Institute of Technology (KIT), Institute for Anthropomatics, Intelligent Sensor-Actuator-Systems Laboratory \\ ${ }^{2}$ Karlsruhe Institute of Technology (KIT), Institute for Anthropomatics, Visions and Fusion Laboratory \\ ${ }^{3}$ Fraunhofer Institute of Optronics, System Technologies and Image Exploitation IOSB \\ Karlsruhe, Germany \\ Email:\{marcus.baum, ioana.gheta, andrey.belkin\}@kit.edu, juergen.beyerer@iosb.fraunhofer.de, uwe.hanebeck@ieee.org
}

\begin{abstract}
This contribution introduces a three pillar information storage and management system for modeling the environment of autonomous systems. The main characteristics is the separation of prior knowledge, environment model and sensor information. In the center of the system is the environment model, which provides the autonomous system with information about the current state of the environment. It consists of instances with attributes and relations as virtual substitutes of entities (persons and objects) of the real world. Important features are the representation of uncertain information by means of Degreeof-Belief (DoB) distributions, the information exchange between the three pillars as well as creation, deletion and update of instances, attributes and relations in the environment model. In this work, a Bayesian method for fusing new observations to the environment model is introduced. For this purpose, a Bayesian data association method is derived. The main question answered here is the observation-to-instance mapping and the decision mechanisms for creating a new instance or updating already existing instances in the environment model.
\end{abstract}

\section{INTRODUCTION}

Autonomous systems acquire information in order to be able to interact with their environment. For intelligent autonomous systems, such as humanoid robots helping with domestic applications, the mere storage and retrieval of information is not enough. Since such an autonomous system has to cope with uncertain and incomplete information about its environment, elaborate information management mechanisms are additionally required.

a) Contributions: First, we propose an intelligent, universal information management system: the three pillar information system. The name of the system is given due to its main characteristic, which is the separation between three components: prior knowledge, environment model, and sensory information. These can be compared to the long and short term memories and the real world. The environment model is the central component of the system and it can be compared with a Lego landscape, where the Lego bricks compose virtual substitutes (instances) of real objects and persons (entities). In the virtual environment, the entities of the real-world object types and persons are represented by instances of classes.

A further contribution is that we introduce a mathematical formalization of the environment model within Bayesian probability theory. This formalization builds the theoretical basis for performing inference in the environment model. All information regarding instances or entities is based on their attributes and relations, which are specified through their uncertainties in form of Degree-of-Belief (DoB) distributions. In this way, information management (creation, deletion, update of instances and their attributes and relations) and exchange in the three pillar information system can be performed within the Bayesian framework.

The third major contribution is to derive formulas for the Bayesian information update in the environment model. Therein, one of the main challenges that appears is data association. It is unknown which instance the new acquired information (new observation regarding the attributes or relations of an entity) regards, or whether it is the result of observing a new entity. Such problems and their implications for the mechanisms of information management are in the focus in this paper. For this purpose, we employ techniques from multi-target tracking, where similar data associations problems appear.

b) Related Work: Modeling the environment of autonomous systems involves modeling information. Common methods for doing so are based on semantic nets, predicate logic or formal languages [1]. Nowadays, the methods involve ontologies, object oriented and probabilistic approaches, and combinations of two of them [2]-[6]. Moreover, the proposed approaches in literature for modeling the environment of autonomous systems are mostly domain-specific and not extendable to other applications. [7] proposes an object-oriented world modeling approach with the purpose of creating virtual environments for simulation or system engineering in the financial domain. [6] proposes a dynamic, object-oriented approach for modeling the relevant environment of cooperative vehicles, incorporating attribute uncertainties. Neither of these contributions deals with data association problems.

Data association is one of the main topics in multi-target tracking [8]. A simple method for data association is the socalled Nearest Neighbor Filter [9] that assigns each observation to the most probable target. The exact Bayesian solution of the data association problem is in general intractable. Hence, approximations like one of the most popular data association techniques called Joint Probabilistic Data Association Filter (JPDAF) [10] have to be employed. The JPDAF can be seen 
as an approximation of the exact Bayesian solution of the data association problem. JPDAF performs a weighted update of all target states according to an association probability. JPDAF ignores correlations between the target states and removes resulting multimodalities in the target state by means of analytic moment matching. JPDAF has also been extended to cope with existence probabilities of tracks [11]. A general Bayesian formulation of multi-target tracking with track existence has been given in [12], [13].

c) Overview: The remainder of this paper is structured as follows: Section II describes the three pillar information system with its characteristics. The information management and data association based on the Bayesian framework are described in Sections III and IV. Section V finally describes an application for the proposed system.

\section{The Three Pillar Information System}

Figure 1 shows the structure of the three pillar information system. The first component represents prior knowledge and incorporates two types of information: instance-related knowledge (e.g., instances of persons or objects, maps) and concepts in form of ontologies (information about classes with attributes, relations, and rules). This information is contextspecific and can be loaded/unloaded as needed, preventing overfitting.

The second and main component is the environment model. It contains dynamic progressive mapping of instances and their attributes and multiple semantic networks for relations. Its main functionality is to act as an information hub for sensors and inference processes [5], [14], [15].

The third component represents the real world with entities of object types and persons with their attributes and relations. Once observed, these are mapped to instances with attributes and relations in the environment model, see Section III. Their equivalents on the side of prior knowledge are instances of concepts.

\section{A. Probabilistic Model for Information}

Each information piece (attribute or relation) is characterized by its uncertainty in form of a DoB distribution. The different ways of expressing uncertainty and the advantages of employing DoB distributions are presented in detail in [5].

An instance $i$ at time step $k$ is therefore modeled as a DoB distribution

$$
p\left(e_{k}^{i}, \underline{a}_{k}^{i}\right)
$$

where $e_{k}^{i} \in\{0,1\}$ specifies whether the instance exists $\left(e_{k}^{i}=\right.$ $1)$, and

$$
\underline{a}_{k}^{i}:=\left[\begin{array}{llll}
t_{k}^{i} & \underline{a}_{k}^{i, 1} & \cdots & \underline{a}_{k}^{i, n_{a}}
\end{array}\right]^{\mathrm{T}}
$$

is a vector that is composed of the discrete type $t_{k}^{i}$ of the instance $i$, and $n_{a}$ (discrete or continuous) descriptive attributes $\underline{a}_{k}^{i, 1}, \ldots, \underline{a}_{k}^{i, n_{a}}$. The attributes of different instances are assumed to be independent (common assumption of JPDAF). Moreover, the type and other descriptive attributes of an instance are assumed to be independent of each other.
Relations are modeled as binary variables and are treated similar to attributes. For a better understanding and reduced complexity, they are not explicitly considered within this contribution.

\section{B. Information Exchange between the Three Components}

Figure 1 shows the main information flows, which mainly deliver and receive information from the environment model.

a) Information Exchange between the Environment Model and Prior Knowledge: Prior knowledge is necessary in the environment model for specification and supplementation of attributes and relations by using ontology information. An important aspect is the specification of the attribute type of an instance, which is equivalent to classification. The rules of ontologies are used for performing validity, relevance, and consistency checks [5]. Instance-related knowledge is mainly used to complement the instances of already observed entities. This increases the efficiency, since the instances in prior knowledge have most attributes already specified.

In the opposite direction, the information flow is equivalent to the process of learning new concepts or just new attributes and relations of already known concepts. It also means long term saving of meaningful instances, e.g., persons that often interact with the autonomous system.

b) Information Exchange between the Environment Model and the World: The information flow from the real world into the environment model is equivalent to the information acquisition process. It consists of initialization, update and deletion of instances (based on the existence attribute), attributes and relations, see Section IV. Therein, the main challenge is data association, i.e., mapping observations to instances, see Section III.

In the opposite direction, the information exchange process is performed with the purpose of filling the gaps (only possible when prior knowledge is incorporated) or (re)confirmation of information (e.g., for lowering the uncertainty).

\section{Building-up the Environment Model}

In the case that new information about an instance is acquired that does not have an equivalent in the environment model, a new instance is created. The decision is based on the computation of the posterior probability that a new entity is detected at time step $k$ and the detected entity exists, which has to exceed an instantiation threshhold

$$
p\left(e_{k}^{i}=1\right)>\gamma_{\mathrm{i}} .
$$

A similar calculus is performed for instantiating attributes or relations, see Section IV-C.

An instance is deleted from the environment model, if the maximum of the DoB distribution for the attribute existence drops below the deletion threshold

$$
p\left(e_{k}^{i}=1\right)<\gamma_{\mathrm{e}}
$$

with $\gamma_{\mathrm{e}}<\gamma_{\mathrm{i}}$. The relation between $\gamma_{\mathrm{e}}$ and $\gamma_{\mathrm{i}}$ is necessary for ensuring a hysteresis, i.e., a created instance should not be immediately deleted, see Figure 2. 


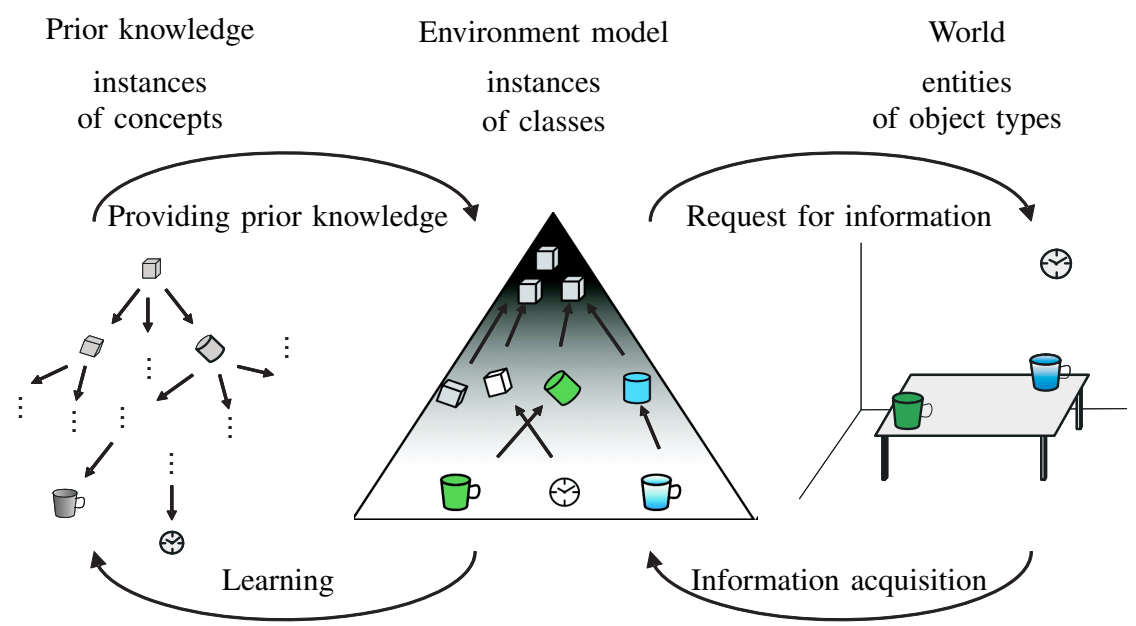

Fig. 1: Three pillar information system.

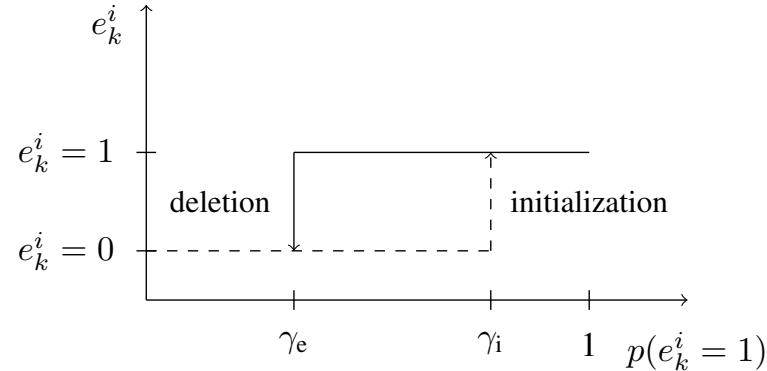

Fig. 2: Hysteresis for the instantiation and the deletion of instances.

If no new information regarding an instance is provided from one time step to the other, a propagation mechanisms alters the DoB distributions according to the maximum entropy principle, i.e., the uncertainty of the information increases, see Section IV-A. If new information is provided, Bayesian fusion of new acquired and old information already stored in the environment model is performed additionally, see Section IV-B.

\section{Data Association: Observation Model}

In order to fuse an observation into the environment model, an observation model that relates the observation to an instance is required. This observation model has to incorporate the uncertainty of the existence of instances and the observationto-instance assignment. Actually, similar problems occur in multi-target tracking [12], [13], where for instance several airplanes are to be tracked by means of radar measurements. There, it is unknown from which target a received measurement stems so that a method for data association is required. Hence, the considered problem here can also be seen as a special multi-target tracking problem. Essentially, the data association mechanism employed in this paper can be seen as a special case of [12], [13].

In the following, we are going to formulate an observation model for the environment model. We assume that at each time step $k$, a single observation is received with regard to a single (unknown) instance. Note that in classical data association for multi-target target tracking one usually deals with a variable number of observations per time step.

The connection between an observation and the instance it regards, is modeled with an association variable $d_{k} \in$ $\left\{0,1 \ldots, N_{k}\right\}$. The probability that the observation at time step $k$ regards the instance $d_{k}$ is given by

$$
p\left(d_{k} \mid \underline{e}_{k}\right),
$$

with $\underline{e}_{k}:=\left[e_{k}^{1}, \ldots, e_{k}^{N_{k}}\right]^{\mathrm{T}}$ and $N_{k}$ as the number of instances in the environment model. An observation that regards a new instance, is represented with $d_{k}=0$.

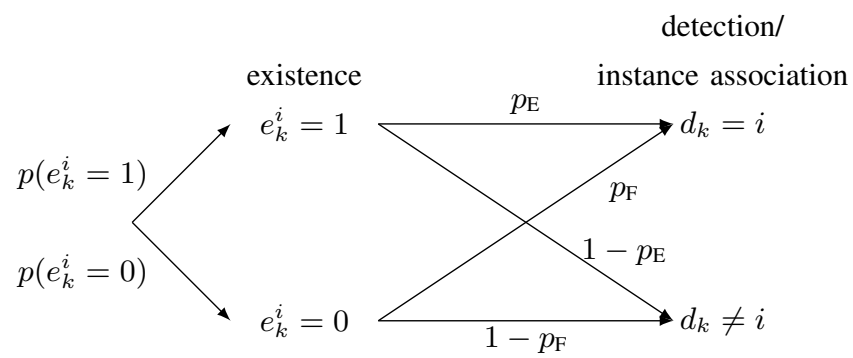

Fig. 3: Schematic dependencies between the existence and observation of entities.

Figure 3 shows the four conditional DoBs regarding the observation and existence of a particular entity $i$ defined [16]:

- The conditional probability $p\left(d_{k}=i \mid e_{k}^{i}=1\right)=p_{\mathrm{E}}$ describes the probability for a hit, i.e., that the entity $i$ exists at the time step $k$ and is observed.

- The conditional probability $p\left(d_{k} \neq i \mid e_{k}^{i}=1\right)=1-p_{\mathrm{E}}$ describes the probability for a miss, i.e., that the entity $i$ exists at the time step $k$, but it is not observed.

- The conditional probability $p\left(d_{k}=i \mid e_{k}^{i}=0\right)=p_{\mathrm{F}}$ describes the probability for a false alarm, i.e., that 
an entity $i$ does not exist at the time step $k$, but an observation is made.

- The conditional probability $p\left(d_{k} \neq i \mid e_{k}^{i}=0\right)=1-p_{\mathrm{F}}$ describes the probability for a correct rejection, i.e., that the entity $i$ does not exist at the time step $k$ and it is not observed.

Next, we need the following instance observation model that gives the probability of an observation, if we know that it regards instance $i$ :

$$
\begin{aligned}
p\left(\underline{y}_{k}^{i} \mid \underline{a}_{k}^{i, 1}, \ldots \underline{a}_{k}^{i, n_{a}}, t_{k}^{i}, e_{k}^{i}, d_{k}=i\right):= \\
p\left(\underline{y}_{k}^{i, 1} \mid \underline{a}_{k}^{i, m_{1}}\right) \cdot \ldots \cdot p\left(\underline{y}_{k}^{i, r} \mid \underline{a}_{k}^{i, m_{r}}\right) \cdot p\left(\mathcal{M}_{k} \mid t_{k}^{i}\right) .
\end{aligned}
$$

Above, $\underline{y}_{k}^{i}:=\left[\underline{y}_{k}^{i, 1}, \ldots, \underline{y}_{k}^{i, r}, \mathcal{M}_{k}\right]^{\mathrm{T}}$ is the observation vector, with $\underline{y}_{k}^{i, 1}, \ldots, \underline{y}_{k}^{i, r}$ as individual observations of particular attributes. The index set of measured attributes is $\mathcal{M}_{k}=\bigcup_{s} m_{s}$. The observation model for an attribute is given by

$$
p\left(\underline{y}_{k}^{i, s} \mid \underline{a}_{k}^{i, m_{s}}\right),
$$

where $m_{s}$ denotes the attribute observed by $\underline{y}_{k}^{i, s}$. The set of observed attributes depends on the particular type of the instance, e.g., for a cup it is likely to measure color and position. Therefore, the conditional DoB $p\left(\mathcal{M}_{k} \mid t_{k}^{i}\right)$ models the DoB of observing the attributes $\mathcal{M}_{k}$, when the type of the instance is given. The DoB $p\left(\mathcal{M}_{k} \mid t_{k}^{i}\right)$ may stem from expert knowledge. It allows also for modeling deterministic information.

The observation model for new, unknown instances is given by the conditional DoB

$$
p\left(\underline{y}_{k}^{i} \mid \underline{a}_{k}^{i, 1}, \ldots \underline{a}_{k}^{i, n_{a}}, t_{k}^{i}, e_{k}^{i}=1, d_{k}=i\right) .
$$

A natuaral choice for this observation model would be

$$
p\left(\underline{y}_{k}^{i} \mid \underline{a}_{k}^{i, 1}, \ldots \underline{a}_{k}^{i, n_{a}}, t_{k}^{i}, e_{k}^{i}=1, d_{k}=i\right):=1 .
$$

However, other definitions may also be justified. For example, a new instance could be more probable to appear on a table than on the floor.

In order to illustrate the observation model for instances, a simple example including entities of type cup is introduced in the following. An instance of type cup may have as attributes color, position, and size. Figure 4 depicts such an instance and a possible observation of a cup entity. A particular sensor for the position of a cup may supply position measurements corrupted by additive Gaussian noise. Hence, a measurement equation for the attribute position could be

$$
\underline{y}_{k}^{i, 5}=\underline{a}_{k}^{i, 5}+v_{k},
$$

where $v_{k}$ denotes additive Gaussian noise. As a consequence, the observation model (4) for the attribute position turns out to be

$$
p\left(\underline{y}_{k}^{i, 5} \mid \underline{a}_{k}^{i, 5}\right)=\mathcal{N}\left(\underline{a}_{k}^{i, 5}-\underline{y}_{k}^{i, 5}, \mathbf{C}_{k}^{v}\right)
$$

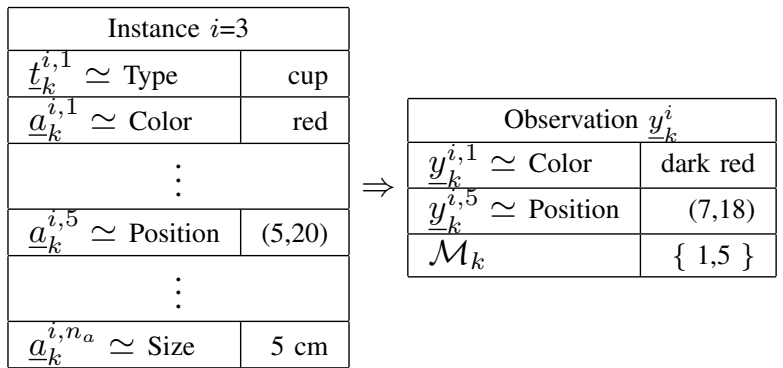

Fig. 4: Example of an instance and an observation.

The attribute observation probability $p\left(\mathcal{M}_{k} \mid t_{k}^{i}=\right.$ cup) may be defined as zero except for

$$
\begin{aligned}
p\left(\{1,5\} \mid t_{k}^{i}=\mathrm{cup}\right) & =0.4, \\
p\left(\{1\} \mid t_{k}^{i}=\mathrm{cup}\right) & =0.3, \\
p\left(\{5\} \mid t_{k}^{i}=\mathrm{cup}\right) & =0.3 .
\end{aligned}
$$

These probabilities say, for example: if the type of the instance is cup, the DoB of observing its position and size is 0.4 .

\section{BAYESIAN UPDATE OF THE ENVIRONMENT MODEL}

This section shows how a Bayesian update of the environment model is performed. The goal is to recursively compute the existence probabilities and the DoB distribution of descriptive attributes for all instances $i$

$$
p\left(e_{k}^{i}=1 \mid \underline{\hat{y}}_{1: k}\right) \quad \text { and } \quad p\left(\underline{a}_{k}^{i} \mid e_{k}^{i}=1, \underline{\hat{y}}_{1: k}\right) .
$$

For this purpose, we employ the common prediction/filtering scheme of Bayesian state estimation.

At each time step $k$, one observation $\hat{y}_{k}$ is received. The data association itself is performed in the filtering step by means of marginalizing over all valid association hypothesis. Note that the complete joint DoB of all instances is not computed. The derivations of the formulas can be seen as a special case of the methods discussed [12], [13].

\section{A. Prediction Step}

The Prediction Step propagates the state of the environment model at time step $k-1$ to the next time step $k$, which results in the predicted existence

$$
p\left(e_{k}^{i}=1 \mid \underline{\hat{y}}_{1: k-1}\right),
$$

and the predicted attributes

$$
p\left(\underline{a}_{k}^{i} \mid e_{k}^{i}=1, \hat{y}_{1: k-1}\right)
$$

for all instances $i$.

The propagation is defined such that the existence probability decreases over time and the entropy of the DoB distribution of attributes and relations increases. The predicted existence probability is achieved by using an exponential decreasing function, which results in the Markov model

$$
\begin{aligned}
& p\left(e_{k}^{i}=1 \mid e_{k-1}^{i}=1\right)=\beta^{i}, \\
& p\left(e_{k}^{i}=1 \mid e_{k-1}^{i}=0\right)=0,
\end{aligned}
$$


where $0<\beta^{i} \leq 1$ is a constant. Hence, we assume that a disappeared instance does not re-appear. Based on this model for existance of an instance, the predicted existence probabilities can be computed according to

$$
p\left(e_{k}^{i}=1 \mid \underline{\hat{y}}_{1: k-1}\right)=\beta^{i} \cdot p\left(e_{k-1}^{i}=1 \mid \underline{\hat{y}}_{1: k-1}\right),
$$

The predicted attribute probabilities result from the Chapman-Kolmogorov equation

$$
\begin{aligned}
& p\left(\underline{a}_{k}^{i} \mid e_{k}^{i}=1, \underline{\hat{y}}_{1: k-1}\right)= \\
& \quad \int p\left(\underline{a}_{k}^{i} \mid \underline{a}_{k-1}^{i}\right) \cdot p\left(\underline{a}_{k-1}^{i} \mid e_{k}^{i}=1, \underline{\hat{y}}_{1: k-1}\right) d \underline{a}_{k-1}^{i}
\end{aligned}
$$

with the dynamic model $p\left(\underline{a}_{k+1}^{i} \mid \underline{a}_{k}^{i}\right)$ for descriptive attributes. If the $\operatorname{DoB} p\left(\underline{a}_{k-1}^{i} \mid e_{k}^{i}=1, \underline{\hat{y}}_{1: k-1}\right)$ is Gaussian and $p\left(\underline{a}_{k}^{i} \mid \underline{a}_{k-1}^{i}\right)$ results from a linear system equation with Gaussian additive noise, the predicted $\operatorname{DoB} p\left(\underline{a}_{k}^{i} \mid e_{k}^{i}=1, \underline{\hat{y}}_{1: k-1}\right)$ is also Gaussian and is computed according to the well-known Kalman filter prediction step [17]. An example for such a dynamic model is a motion model which specifies the temporal evolution of the position of an instance.

\section{B. Filter Step}

The Filter Step takes the predicted state for time step $k$ and the next observation $\hat{y}_{k}$ in order to compute (5) according to Bayes' rule [18]. As already mentioned, the filter step actually performs the data association. In the following, we derive the detailed formulas for the filter step.

The updated probabilities for attributes can be written according to the Law Of Total Probability as a mixture

$$
\begin{aligned}
p\left(\underline{a}_{k}^{i} \mid e_{k}^{i}=1, \underline{\hat{y}}_{1: k}\right) & = \\
\sum_{d_{k}} p\left(\underline{a}_{k}^{i} \mid e_{k}^{i}\right. & \left.=1, \underline{\hat{y}}_{1: k}, d_{k}\right) \cdot p\left(d_{k} \mid e_{k}^{i}=1, \underline{\hat{y}}_{1: k}\right),
\end{aligned}
$$

where the first term is the posterior distribution for a given association

$$
\begin{aligned}
p\left(\underline{a}_{k}^{i} \mid e_{k}^{i}=1,\right. & \left.\underline{\hat{y}}_{1: k}, d_{k}\right) \propto \\
& p\left(\underline{\hat{y}}_{k}^{d_{k}} \mid e_{k}^{i}=1, \underline{a}_{k}^{i}\right) \cdot p\left(\underline{a}_{k}^{i} \mid e_{k}^{i}=1, d_{k}, \underline{\hat{y}}_{1: k-1}\right)
\end{aligned}
$$

and can be computed with the measurement model for instances (4) and the predicted attribute probabilities (7). In case of a continuous attribute represented with a Gaussian DoB and a linear measurement model, the Kalman filter equation [17] can be employed to computed this posterior DoB. The second term in (8) can be seen as a weighting factor, which specifies the probability that the measurement stems from instance $d_{k}$.

The updated probability for the existence can be written as

$$
p\left(e_{k}^{i}=1 \mid \underline{\hat{y}}_{1: k}\right)=\sum_{d_{k}} p\left(e_{k}^{i}=1, d_{k} \mid \underline{\hat{y}}_{1: k}\right) .
$$

In order to compute $p\left(e_{k}^{i}=1, d_{k} \mid \hat{y}_{1: k}\right)$ in (9) and $p\left(d_{k} \mid e_{k}^{i}=\right.$ $\left.1, \underline{\hat{y}}_{1: k}\right)$ in (8), we need the joint DoB of the updated existence and association variable

$$
p\left(\underline{e}_{k}, d_{k} \mid \underline{\hat{y}}_{1: k}\right) \propto p\left(\underline{\hat{y}}_{k} \mid \underline{e}_{k}, d_{k}, \underline{\hat{y}}_{1: k-1}\right) \cdot p\left(\underline{e}_{k}, d_{k} \mid \underline{\hat{y}}_{1: k-1}\right)
$$

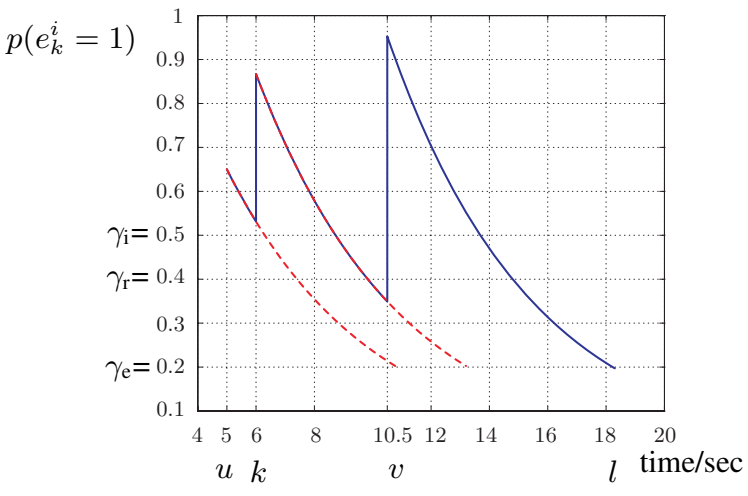

Fig. 5: Example of the life-cycle of an instance. The red dashed line describes the propagation resulting from the aging mechanism, if no new observation is available. The continuous line describes the course of the DoBs when the existence of the entity is reconfirmed at the time steps $k=6$ and $v=10.5$.

where

$$
\begin{aligned}
& p\left(\underline{\hat{y}}_{k} \mid \underline{e}_{k}, d_{k}, \underline{\hat{y}}_{1: k-1}\right)= \\
& \quad \int p\left(\underline{\hat{y}}_{k}^{d_{k}} \mid e_{k}^{i}, \underline{a}_{k}^{i}, d_{k}, \underline{\hat{y}}_{1: k-1}\right) \cdot p\left(\underline{a}_{k}^{i} \mid e_{k}^{i}, d_{k}, \underline{\hat{y}}_{1: k-1}\right) d \underline{a}_{k}^{i}
\end{aligned}
$$

and

$$
p\left(\underline{e}_{k}, d_{k} \mid \underline{\hat{y}}_{1: k-1}\right)=p\left(d_{k} \mid \underline{e}_{k}\right) \cdot p\left(\underline{e}_{k} \mid \underline{\hat{y}}_{1: k-1}\right),
$$

is the product of the association prior (3) and the predicted existence DoB (6).

Note that the DoB (8) is a mixture DoB in the case of continuous distributions. In this case some kind of complexity reduction has to be performed. If the DoB (8) is a Gaussian mixture, it can be collapsed into one Gaussian distribution by means of analytic moment matching similar to JPDAF [10].

Furthermore, with our approach it is not necessary to perform gating, because only one measurement at each time step is received. The only reason to introduce a gating procedure is to reduce the complexity of continuous distributions.

\section{Dealing with an Unknown Number of Instances}

Since the number of instances $N_{k}$ in the environment model may vary, a mechanism that decides how many instances are to be tracked is needed.

If the probability that the observation $\underline{\hat{y}}_{k}$ stems from a new instance exceeds a threshold $\gamma_{\mathrm{e}}$, i.e.,

$$
p\left(d_{k}=0 \mid e_{k}^{0}=1, \underline{\hat{y}}_{1: k}\right)>\gamma_{\mathrm{e}},
$$

the observation cannot be assigned to one of the existing instances

$$
\left[\begin{array}{ll}
e_{k}^{1} & \underline{a}_{k}^{1}
\end{array}\right], \ldots,\left[\begin{array}{ll}
e_{k}^{N_{k}} & \underline{a}_{k}^{N_{k}}
\end{array}\right] .
$$

As a consequence, a new possible instance

$$
\left[e_{k}^{N_{k}+1} \underline{a}_{k}^{N_{k}+1}\right]
$$

is initialized with a proper prior DoB for the existence, and attributes. If in fact this new instance exists, its existence has 


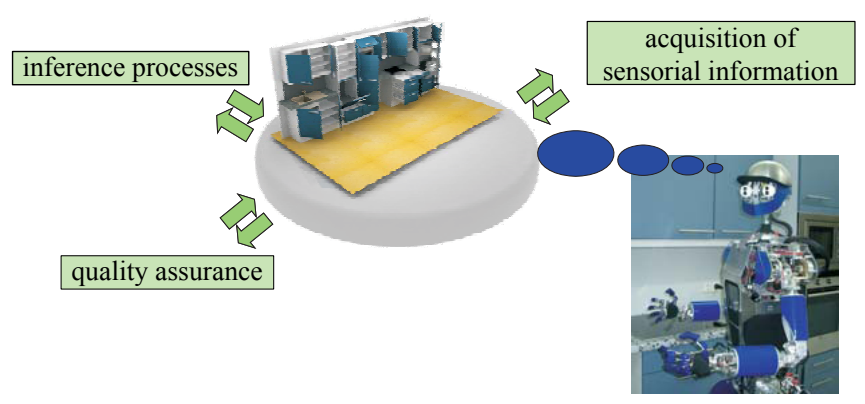

Fig. 6: Environment model for a humanoid robot.

to be confirmed with the help of subsequent measurements: If the $\mathrm{DoB}$ of the existence exceeds a given threshold, the existence is confirmed (see Equation (1)). On the other hand, an instance $i$ is deleted from the environment model when its existence is improbable, i.e., the probability of its existence drops below a threshold (see Equation (2)). Since, we assume that a disappeared instance cannot reappear, we can stop propagating its existence.

Figure 5 shows a typical example of the life-cycle of an instance according to the maximum of the DoB distribution for the attribute existence.

\section{ApPlication}

One of the applications of the three pillar information system is for the DFG Collaborative Research Center (Sonderforschungsbereich) SFB 588 "Humanoid Robots-Learning

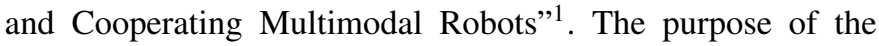
project is to build a humanoid robot [19], [20] helping with domestic applications. The developed test environment is a kitchen, where the robot accomplishes tasks such as set the table or helping with cooking. The robot also can operate kitchen appliances like dish washer or fridge, see Figure 6. Details of development and implementation as well as a practical example are given in [14], [15].

\section{CONClusions AND FUtURE WORK}

In this work, a three pillar information storage and management system for modeling the environment of autonomous systems was introduced. The main characteristics of this system is the separation of prior knowledge, environment model and sensory information. Furthermore, since an autonomous system typically acts in an uncertain environment, an important feature is the representation of uncertain information by means of Degree-of-Belief (DoB) distributions. In this contribution we have focused on fusing new observations with the environment model. We introduced a method for dealing with unknown observation-to-instance mappings and a decision mechanism for creating new instances or updating already existing ones in the environment model. For this purpose, we have employed techniques from Bayesian data association in the field of target tracking.
Future work includes a detailed evaluation of the introduced data association mechanism for the three pillar information storage and management system in a real-world scenario. Furthermore, future work will be concerned with solving the classification problem, i.e., mapping of instances from the environment model to concepts from prior knowledge. The challenges involve the integration of semantic knowledge, e.g., about attributes and the search for appropriate methods for comparing DoB distributions.

\section{REFERENCES}

[1] P. Russell, Stuart J. ; Norvig, Artificial intelligence, 3rd ed. Pearson Education, 2010.

[2] A. Bauer, "Probabilistic Reasoning on Object Occurrence in Complex Scenes," in Image and Signal Processing for Remote Sensing XV, Proc. of SPIE, vol. 7477, 2009.

[3] P. C. G. da Costa, K. B. Laskey, and K. J. Laskey, "PR-OWL: A Bayesian Ontology Language for the Semantic Web," in ISWC-URSW, 2005, pp. 23-33.

[4] W. V. Siricharoen, "Ontologies and Object models in Object Oriented Software Engineering," IAENG International Journal of Computer Science, vol. 33, no. 1, 2007.

[5] I. Gheţa, M. Heizmann, and J. Beyerer, "Object Oriented Environment Model for Autonomous Systems," in Proceedings of the second Skövde Workshop on Information Fusion Topics, H. Boström, R. Johansson, and J. van Laere, Eds. Skövde Studies in Informatics, Nov. 2008, pp. 9-12.

[6] Z. Papp, C. Brown, and C. Bartels, "World Modeling for Cooperative Intelligent Vehicles," in IEEE Intelligent Vehicles Symposium, 2008, pp. $1050-1055$.

[7] S. Isoda, "Object-Oriented World-Modeling Revisited," Journal of Systems and Software, vol. 59, no. 2, pp. 153-162, 2001.

[8] D. Hall and J. Linas, Handbook of Multisensor Data Fusion. CRC Press, May 2001.

[9] X. Rong Li and Y. Bar-Shalom, "Tracking in clutter with nearest neighbor filters: analysis and performance," Aerospace and Electronic Systems, IEEE Transactions on, vol. 32, no. 3, pp. 995 -1010, july 1996.

[10] Y. Bar-Shalom, F. Daum, and J. Huang, "The Probabilistic Data Association Filters," Control Systems Magazine, IEEE, vol. 29, no. 6, pp. 82 -100 , Dec. 2009.

[11] D. Musicki and R. Evans, "Joint Integrated Probabilistic Data Association - JIPDA," vol. 2, 2002, pp. 1120 - 1125 vol.2.

[12] J. Vermaak, S. Maskell, and M. Briers, "A Unifying Framework for Multi-Target Tracking and Existence," vol. 1, July 2005.

[13] P. Horridge and S. Maskell, "Searching for, Initiating and Tracking Multiple Targets using Existence Probabilities," July 2009, pp. 611 617.

[14] A. Belkin, "Object-Oriented World Modeling for Autonomous Systems," Karlsruhe Institute of Technology KIT, Tech. Rep., 2010.

[15] B. Kühn, A. Belkin, A. Swerdlow, T. Machmer, J. Beyerer, and K. Kroschel, "Knowledge-Driven Opto-Acoustic Scene Analysis based on an Object-Oriented World Modelling Approach for Humanoid Robots," in Proceedings of the 41st International Symposium on Robotics and the 6th German Conference on Robotics. VDE-Verlag, 2010.

[16] R. O. Duda, P. E. Hart, and D. G. Stork, Pattern Classification. John Wiley \& Sons Inc, 2004.

[17] D. Simon, Optimal State Estimation: Kalman, H Infinity, and Nonlinear Approaches, 1st ed. Wiley \& Sons, August 2006.

[18] J. M. Bernardo, Encyclopedia of Life Support Systems (EOLSS). Probability and Statistics. Oxford: UNESCO, 2003, ch. Bayesian Statistics.

[19] T. Asfour, K. Regenstein, P. Azad, J. Schroder, A. Bierbaum, N. Vahrenkamp, and R. Dillmann, "ARMAR-III: An Integrated Humanoid Platform for Sensory-Motor Control," in 6th IEEE-RAS International Conference on Humanoid Robots, 2006, pp. 169-175.

[20] T. Asfour, P. Azad, N. Vahrenkamp, K. Regenstein, A. Bierbaum, K. Welke, J. Schröder, and R. Dillmann, "Toward Humanoid Manipulation in Human-Centred Environments," Robot. Auton. Syst., vol. 56, no. 1 , pp. 54-65, 2008.

\footnotetext{
${ }^{1}$ http://www.sfb588.uni-karlsruhe.de
} 